\title{
On Lindenstrauss-Pełczyński spaces
}

\author{
by \\ Jesús Castillo, Yolanda Moreno and Jesús Suárez (Badajoz)
}

\begin{abstract}
We consider some stability aspects of the classical problem of extension of $C(K)$-valued operators. We introduce the class $\mathcal{L} \mathcal{P}$ of Banach spaces of LindenstraussPełczyński type as those such that every operator from a subspace of $c_{0}$ into them can be extended to $c_{0}$. We show that all $\mathcal{L} \mathcal{P}$-spaces are of type $\mathcal{L}_{\infty}$ but not conversely. Moreover, $\mathcal{L}_{\infty}$-spaces will be characterized as those spaces $E$ such that $E$-valued operators from $w^{*}\left(l_{1}, c_{0}\right)$-closed subspaces of $l_{1}$ extend to $l_{1}$. Regarding examples we will show that every separable $\mathcal{L}_{\infty}$-space is a quotient of two $\mathcal{L} \mathcal{P}$-spaces; also, $\mathcal{L}_{\infty}$-spaces not containing $c_{0}$ are $\mathcal{L} \mathcal{P}$-spaces; the complemented subspaces of $C(K)$ and the separably injective spaces are subclasses of the $\mathcal{L} \mathcal{P}$-spaces and we show that the former does not contain the latter. Regarding stability properties, we prove that quotients of an $\mathcal{L} \mathcal{P}$-space by a separably injective space and twisted sums of $\mathcal{L} \mathcal{P}$-spaces are $\mathcal{L} \mathcal{P}$-spaces.
\end{abstract}

1. Introduction and preliminaries. In this work we shall be concerned with some stability aspects of the classical problem of extension of $C(K)$-valued and $\mathcal{L}_{\infty}$-valued operators. Let us describe and motivate them. In a 1971 paper [26] Lindenstrauss and Pełczyński proved:

THEOREM 1. Let $K$ be a compact Hausdorff space. Every $C(K)$-valued operator defined on a subspace of $c_{0}$ admits an extension to the whole space.

The result remained isolated until 1989 when Johnson and Zippin obtained in [17] an extension to subspaces of $c_{0}(\Gamma)$, and later in 1995, in [18], the analogous result for $w\left(l_{1}, c_{0}\right)$-closed subspaces of $l_{1}$. Further proofs of the Lindenstrauss-Pełczyński theorem have been provided by Zippin [34, 35]. The paper [8] contains a homological approach to both results showing that they are in a sense dual to each other.

The general problem of extension of operators admits a natural formulation in homological terms. We shall assume from the reader some familiarity

2000 Mathematics Subject Classification: 46B20, 46M18, 46B25.

Key words and phrases: extension of operators, exact sequence of Banach spaces, 3 -space property, operator ideal.

The research of the first two authors has been supported in part by DGICYT project MTM2004-02635. The work of the third author was supported in part by a Marie Curie grant HPMT-GH-01-00286-04 at Karlsruhe University under the direction of Prof. L. Weis. 
with the basic notions and constructions of the theory of exact sequences of Banach spaces; the necessary background can be found in [9] and, operatively defined, below. We shall write $0 \rightarrow Y \stackrel{j}{\rightarrow} X \stackrel{q}{\rightarrow} Z \rightarrow 0 \equiv F$ to represent an exact sequence of Banach spaces and operators, which is a diagram where the kernel of each operator coincides with the image of the preceding one. The open mapping theorem makes $Y$ a subspace of $X$ through the embed$\operatorname{ding} j$ and $Z$ the corresponding quotient space through $q$. The reader can view $F$ just as the name of the sequence; however, those familiar with the theory of quasi-linear maps created in $[19,22]$ can in fact consider $F$ as a quasi-linear map associated to the exact sequence.

We shall consider exact sequences of Banach spaces modulo the natural equivalence relation: two sequences $F$ and $G$ are said to be equivalent if there is a commutative diagram

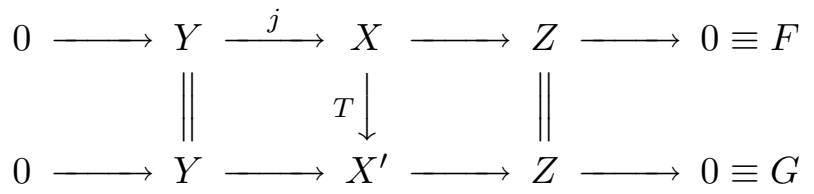

In this case we write $F \equiv G$. The space of equivalence classes of exact sequences with $Y$ as subspace and $Z$ as quotient will be denoted $\operatorname{Ext}(Z, Y)$. It is a vector space under some natural operations (see [13, III.2]) and the 0 element is the sequence $0 \rightarrow Y \rightarrow Y \oplus Z \rightarrow Z \rightarrow 0$ with inclusion $y \mapsto(y, 0)$ and quotient map $(y, z) \mapsto z$. We shall say that $F$ is trivial or splits when $F \equiv 0$. This means, in classical terms, that $j(Y)$ is complemented in $X$. Recall that a property $\mathcal{P}$ is said to be a 3-space property if whenever one has an exact sequence $0 \rightarrow Y \rightarrow X \rightarrow Z \rightarrow 0$ in which both $Y$ and $Z$ have $\mathcal{P}$ then also $X$ has $\mathcal{P}$; see [9] for most of the available information about 3 -space problems.

The lower sequence in a diagram

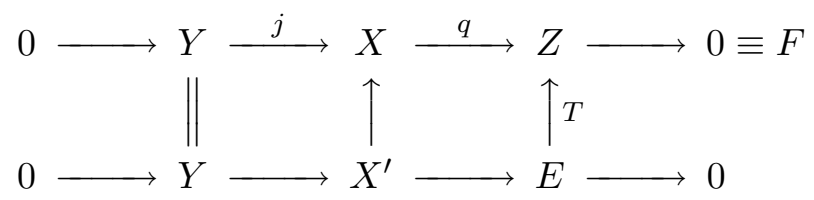

is a called a pull-back sequence and is naturally denoted FT. The middle space $X^{\prime}$ is called the pull-back of $q$ and $T$. The sequence $F T$ splits if and only if $T$ can be lifted to $X$ through $q$. The lower sequence in a diagram

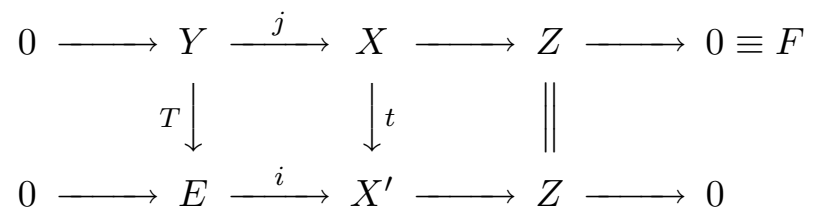


is called the push-out sequence and is naturally denoted $T F$. The middle space $X^{\prime}$ is called the push-out of $j$ and $T$. This space has the universal property that given operators $a: E \rightarrow B$ and $b: X \rightarrow B$ such that $a T=b j$ there exists a unique operator $p: X^{\prime} \rightarrow B$ such that $p i=a$ and $p t=b$. Extending an operator $T: Y \rightarrow E$ through $j$ is the same as saying that $T F$ is trivial. The lifting property of $l_{1}(\Gamma)$ and the fact that every Banach space $Z$ admits an exact sequence $0 \rightarrow K(Z) \rightarrow l_{1}(\Gamma) \rightarrow Z \rightarrow 0$, called a projective presentation of $Z$, imply that every exact sequence $0 \rightarrow Y \rightarrow X \rightarrow Z \rightarrow 0$ is a push-out of a projective presentation of $Z$. Hence $\operatorname{Ext}(Z, Y)=0$ is equivalent to the statement "every operator $K(Z) \rightarrow Y$ can be extended to $l_{1}(\Gamma)$ ".

That all operators $Y \rightarrow E$ can be extended to $X$ through $j$ admits an even simpler formulation: the restriction operator $j^{*}: \mathcal{L}(X, E) \rightarrow \mathcal{L}(Y, E)$ is surjective. The following terminology is quite natural and will prove to be very useful:

Definition 1. Let $\mathcal{A}$ be a class of Banach spaces. We say that an exact sequence $0 \rightarrow Y \stackrel{j}{\rightarrow} X \rightarrow Z \rightarrow 0 \equiv F$ is $\mathcal{A}$-trivial (or that $F \mathcal{A}$-splits) if for every $A \in \mathcal{A}$ the restriction operator $j^{*}: \mathcal{L}(X, A) \rightarrow \mathcal{L}(Y, A)$ is surjective. We will also say that $Y$ is $\mathcal{A}$-complemented in $X$.

Sometimes the quantitative version of the previous notion will be used: given $\lambda \geq 1$, the exact sequence $F$ will be said to be $(\lambda, \mathcal{A})$-trivial if for every $A \in \mathcal{A}$ every operator $T: Y \rightarrow A$ admits an extension $\widehat{T}: X \rightarrow A$ such that $\|\widehat{T}\| \leq \lambda\|T\|$.

This notion of $\mathcal{A}$-triviality unifies different notions appearing in the literature: (i) trivial sequences, which correspond to $\mathcal{A}=$ all Banach spaces; (ii) Kalton's locally trivial, or locally split, sequences (see [20]), corresponding to $\mathcal{A}=l_{\infty}\left(G_{n}\right)$, where $G_{n}$ is a dense (in the Banach-Mazur distance) sequence of finite-dimensional Banach spaces (see also [14]); (iii) Zippin's almost trivial sequences (see [33-36]), which correspond to the choice $\mathcal{A}=$ $C(K)$-spaces.

In this work we are concerned with $C(K)$-trivial and $\mathcal{L}_{\infty}$-trivial sequences. In Section 2 we study the stability of $C(K)$-trivial sequences by amalgams and duality. We first show that $l_{p^{-}}$and $c_{0^{-}}$amalgams of $C(K)$ trivial sequences are $C(K)$-trivial. Concerning the stability of $C(K)$-trivial sequences by duality, the Lindenstrauss-Pełczyński and Johnson-Zippin theorems suggest that it could be that the dual of a $C(K)$-trivial sequence is $C(K)$-trivial since the former implies that every exact sequence $0 \rightarrow H \rightarrow$ $X \rightarrow S \rightarrow 0 \equiv F$ with $H$ a subspace of $c_{0}$ and $S$ separable is $C(K)$-trivial; and the latter yields (see [10]) that its dual sequence $F^{*}$ is $C(K)$-trivial. However, the situation covered by those two theorems proves to be quite peculiar; we give examples at the end of Section 2 to show that the dual and bidual sequences of a $C(K)$-trivial sequence need not be $C(K)$-trivial. 
In Section 3 our attention turns to those Banach spaces which can play the role of $C(K)$-spaces in the Lindenstrauss-Pełczyński theorem. We call such spaces Lindenstrauss-Pełczyński $(\mathcal{L} \mathcal{P}$, for short) spaces. Our motivation to introduce those spaces comes from [26, p. 234, remark 2] where Lindenstrauss and Pełczyński assert that isometric $L_{1}$-preduals can play the role of $C(K)$-spaces regarding extension of operators from subspaces of $c_{0}$. After showing that every $\mathcal{L} \mathcal{P}$-space is an $\mathcal{L}_{\infty}$-space we then face the unavoidable question: Must every $\mathcal{L}_{\infty}$-space be an $\mathcal{L} \mathcal{P}$-space? The answer is no, which solves Problem 6.15 of Zippin in [36]. We shall show that the same approach to the Johnson-Zippin theorem just provides a new characterization of $\mathcal{L}_{\infty}$-spaces (see Proposition 3.1).

As regards the problem of the identification of $\mathcal{L} \mathcal{P}$-spaces, it is clear that complemented subspaces of $C(K)$-spaces and separably injective spaces are $\mathcal{L} \mathcal{P}$-spaces. On the way, we will give perhaps the first example of a separably injective space that is not complemented in any $C(K)$-space. The previous examples do not exhaust the class of $\mathcal{L} \mathcal{P}$-spaces: we shall show that $\mathcal{L}_{\infty}$-spaces not containing $c_{0}$, the new exotic $\mathcal{L}_{\infty}$-spaces constructed in [7], quotients of $\mathcal{L} \mathcal{P}$-spaces by separably injective subspaces and $c_{0}$-vector sums of uniformly $\mathcal{L} \mathcal{P}$-spaces are $\mathcal{L} \mathcal{P}$-spaces.

In Section 4 we tackle the 3 -space problem for the class of $\mathcal{L} \mathcal{P}$-spaces, which needs the development of a new method of proof and new characterizations of $\mathcal{L} \mathcal{P}$-spaces. Section 5 contains further remarks, examples and open problems.

2. On the stability of $C(K)$-trivial sequences by amalgams and duality. To study the stability of $C(K)$-trivial sequences we need to know their behavior with respect to the basic homological pull-back and push-out constructions.

Proposition 2.1. Let $\mathcal{A}$ be a class of Banach spaces.

(1) A pull-back sequence of an $\mathcal{A}$-trivial sequence is $\mathcal{A}$-trivial.

(2) A push-out sequence of an $\mathcal{A}$-trivial sequence is $\mathcal{A}$-trivial.

Proof. The first assertion is obvious. The second is a consequence of the universal property of the push-out construction.

When, moreover, the push-out is obtained from a surjective operator one has:

Lemma 1. Let $\mathcal{A}$ be a class of Banach spaces. Consider the completed push-out diagram of Banach spaces 


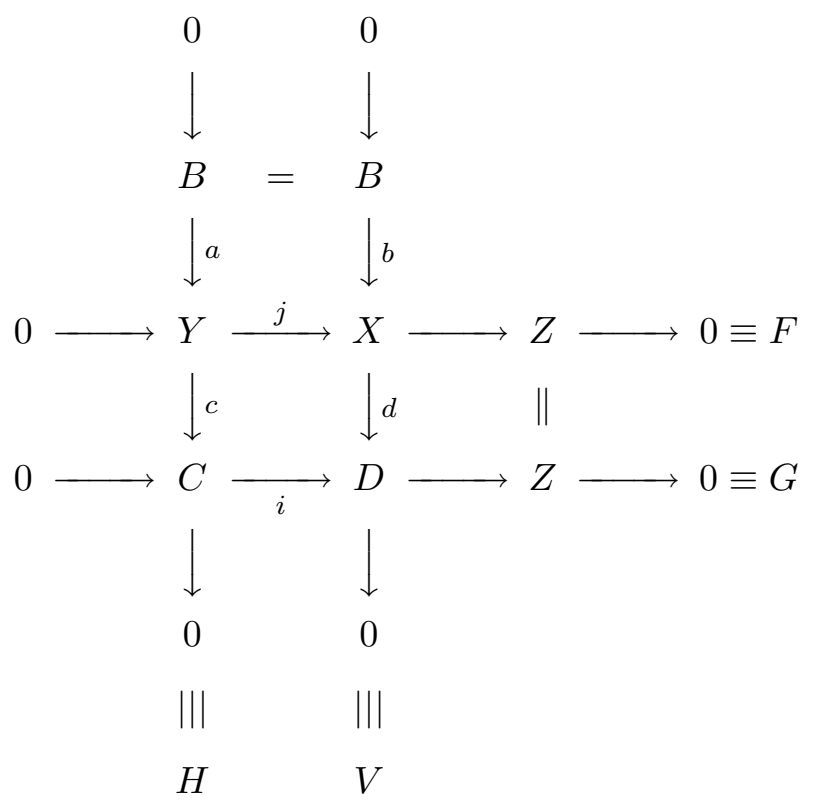

(i) $V$ and $G$ are $\mathcal{A}$-trivial if and only if $F$ and $H$ are $\mathcal{A}$-trivial.

(ii) If $F$ is $\mathcal{A}$-trivial then $G$ is $\mathcal{A}$-trivial; if $V$ is $\mathcal{A}$-trivial, $H$ is $\mathcal{A}$-trivial.

Proof. The second part of (ii) follows from Proposition 2.1(1). Let $A \in \mathcal{A}$ and notice that from the diagram in the hypothesis we can construct the commutative diagram

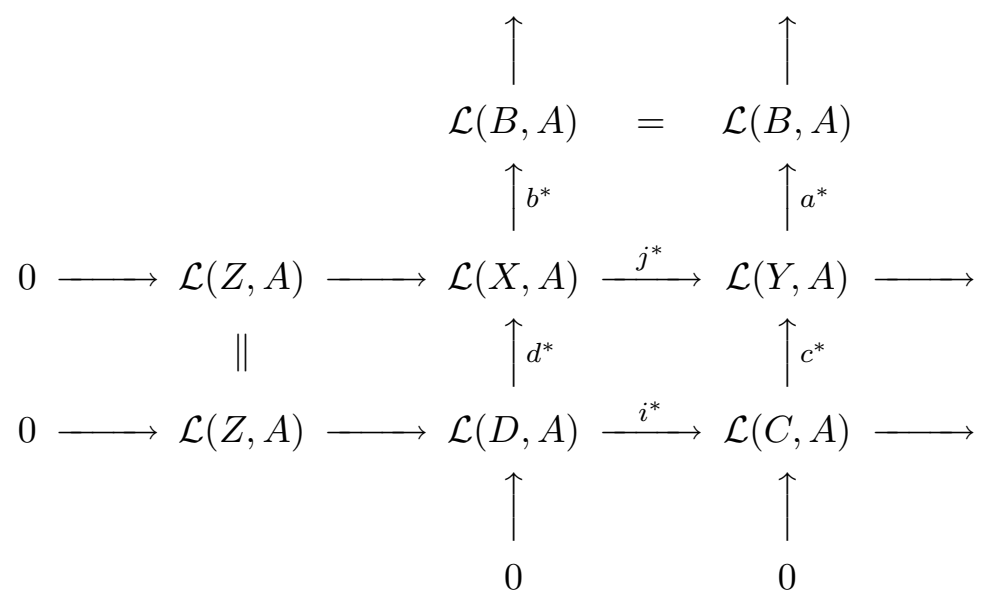

Now, the first part of (ii) can be easily obtained by diagram chasing while (i) follows by simply observing that the restriction operators $b^{*}$ and $i^{*}$ are surjective if and only if $j^{*}$ and $a^{*}$ are surjective.

Remark. Pełczyński's Proposition 2.6 of [30] can be considered a rudimentary version of this principle. 
The characterization of $C(K)$-trivial extensions that Zippin formulates and proves in [33] is especially interesting for us.

Lemma 2. A sequence $0 \rightarrow Y \stackrel{j}{\rightarrow} X \rightarrow Z \rightarrow 0$ is $(\lambda, C(K))$-trivial if and only if there is a $w^{*}$-continuous map $\omega: B_{Y^{*}} \rightarrow \lambda B_{X^{*}}$ such that $j^{*} \omega=\mathrm{id}$.

The map $\omega$ will be called a $\lambda$ - $w^{*}$-selector for $j^{*}$. Zippin $[34,35]$ uses this criterion to obtain different proofs of the Lindenstrauss-Pełczyński theorem. It is inspired by the most natural possible situation: the sequence $0 \rightarrow Y \stackrel{\delta_{Y}}{\rightarrow}$ $C\left(B_{Y^{*}}\right) \rightarrow C\left(B_{Y^{*}}\right) / Y \rightarrow 0 \equiv \complement_{Y}$, in which $\delta_{Y}: Y \rightarrow C\left(B_{Y^{*}}\right)$ is the canonical embedding, is $(1, C(K))$-trivial; indeed, the map $\omega: B_{Y^{*}} \rightarrow B_{C\left(B_{Y^{*}}\right)}$ defined as $\omega\left(x^{*}\right)(f)=f\left(x^{*}\right)$ is a $1-w^{*}$-selector for $\delta_{Y}^{*}$. Let us remark that every $C(K)$-trivial sequence is a pull-back of $\complement_{Y}$ and conversely.

It will be useful to notice that some properties of the $w^{*}$-topology in $l_{p}$, $1 \leq p \leq \infty$, pass to $l_{p}$-vector sums of Banach spaces. Given an $l_{p^{-}}$sum $l_{p}\left(X_{n}\right)$ we denote by $\pi_{j}: l_{p}\left(X_{n}\right) \rightarrow X_{j}$ the natural projections. It is straightforward that given a sequence $\left(E_{n}^{*}\right)_{n}$ of dual spaces, a bounded net $\left(x_{\alpha}\right)_{\alpha}$ in $l_{p}\left(E_{n}^{*}\right)$, $1 \leq p \leq \infty$, is $w^{*}$-null if and only if for each $j$ the net $\left(\pi_{j}\left(x_{\alpha}\right)\right)_{\alpha}$ is $w^{*}$-null.

Given a family of exact sequences $0 \rightarrow A_{n} \rightarrow B_{n} \rightarrow C_{n} \rightarrow 0 \equiv F_{n}$, we call $0 \rightarrow l_{p}\left(A_{n}\right) \rightarrow l_{p}\left(B_{n}\right) \rightarrow l_{p}\left(C_{n}\right) \rightarrow 0 \equiv l_{p}\left(F_{n}\right)$ the $l_{p^{-}}$amalgam of $\left(F_{n}\right)_{n}$, for $1 \leq p \leq \infty$. Analogously, the $c_{0}$-amalgam of $\left(F_{n}\right)$ will be denoted $c_{0}\left(F_{n}\right)$. One has:

Proposition 2.2. The $c_{0}$ - and $l_{p}$-amalgams of $(\lambda, C(K))$-trivial exact sequences, $1 \leq p<\infty$, are $(\lambda, C(K))$-trivial.

Proof. For each $n$, let $\omega_{n}: B_{A_{n}^{*}} \rightarrow \lambda B_{B_{n}^{*}}$ be a $\lambda-w^{*}$-selector for $j_{n}^{*}$. If we have the $l_{p}$-amalgam

$$
0 \rightarrow l_{p}\left(A_{n}\right) \stackrel{\chi}{\rightarrow} l_{p}\left(B_{n}\right) \rightarrow l_{p}\left(C_{n}\right) \rightarrow 0 \equiv l_{p}\left(F_{n}\right),
$$

it follows from the observation above that the map $\Omega: B_{l_{p^{*}}\left(A_{n}^{*}\right)} \rightarrow \lambda B_{l_{p^{*}}\left(B_{n}^{*}\right)}$ defined by $\Omega\left[\left(a_{n}^{*}\right)\right]=\left[\omega_{n}\left(a_{n}^{*}\right)\right]$ is a $\lambda$-w $w^{*}$-selector for $\chi^{*}$.

The situation for $l_{\infty}$-amalgams is entirely different because a subspace $X$ of $l_{\infty}$ can only be $C(K)$-complemented if $X$ enjoys both the DunfordPettis property (weakly compact operators transform weakly compact sets into relatively compact sets) and the Grothendieck property (all operators with separable range are weakly compact): Indeed, if $E$ is separable and $\tau: X \rightarrow E$ is an operator, then $\delta_{E} \tau: X \stackrel{\tau}{\rightarrow} E \stackrel{\delta_{E}}{\rightarrow} C\left(B_{E^{*}}\right)$ should extend to an operator $l_{\infty} \rightarrow C\left(B_{E^{*}}\right)$; by the Grothendieck property of $l_{\infty}$ (see $\left[12\right.$, Cor. 12 , p. 156]) this operator is weakly compact, hence $\delta_{E} \tau$ is weakly compact, as also is $\tau$. We have shown that $X$ has the Grothendieck property. Since $l_{\infty}$ has the Dunford-Pettis property, every weakly compact operator $X \rightarrow c_{0}$ must do the same, which means that $X$ also enjoys the Dunford- 
Pettis property. In particular, subspaces of $l_{\infty}$ such as $l_{\infty}\left(l_{2}^{n}\right)$, which does not have the Dunford-Pettis property because it contains a complemented copy of $l_{2}$, provide sequences $0 \rightarrow l_{\infty}\left(l_{2}^{n}\right) \rightarrow l_{\infty} \rightarrow Q \rightarrow 0$ that are not $C(K)$-trivial. Therefore, the $l_{\infty}$-amalgam of the sequences

$$
0 \rightarrow l_{2}^{n} \rightarrow l_{\infty}^{m(n)} \rightarrow l_{\infty}^{m(n)} / l_{2}^{n} \rightarrow 0
$$

in which the embeddings are $(1+\varepsilon)$-isometries cannot be $C(K)$-trivial. Nevertheless, those sequences are all $\left((1+\varepsilon)^{2}, C(K)\right)$-trivial by the existence of the Bartle-Graves continuous selection.

Later on we shall also show that the $c_{0}$-amalgam of $\mathcal{L}_{\infty}$-trivial sequences is not necessarily $\mathcal{L}_{\infty}$-trivial. Our concern now is to study the stability of $C(K)$-trivial sequences by duality. Let us start by observing that, for every subspace $H$ of $c_{0}$ and every separable Banach space $S$, the sequence $0 \rightarrow$ $H \rightarrow X \rightarrow S \rightarrow 0 \equiv F$ is $C(K)$-trivial as is its dual $F^{*}$. That $F$ is $C(K)$ trivial was observed by Lindenstrauss and Pełczyński in [26, Cor. 2]. The assertion about $F^{*}$ (actually, that every exact sequence $0 \rightarrow Y \rightarrow X \rightarrow$ $H^{*} \rightarrow 0$ is $C(K)$-trivial for every subspace $H$ of $c_{0}$ ) directly follows from the equality $\operatorname{Ext}\left(H^{*}, C(K)\right)=0$, which is a consequence of the Johnson-Zippin theorem. It is not true, in general, that the dual or bidual of a $C(K)$-trivial sequence is $C(K)$-trivial:

EXAMPLES. Consider the $C(K)$-trivial sequence $0 \rightarrow l_{2} \stackrel{\delta}{\rightarrow} C\left(B_{l_{2}}\right) \rightarrow$ $Q \rightarrow 0 \equiv F$. We claim that the dual sequence $0 \rightarrow Q^{*} \rightarrow L_{1} \stackrel{\delta^{*}}{\rightarrow} l_{2} \rightarrow 0 \equiv F^{*}$ is not $C(K)$-trivial. Assume otherwise. Consider a projective presentation of $l_{2}$,

$$
0 \rightarrow K\left(l_{2}\right) \rightarrow l_{1} \rightarrow l_{2} \rightarrow 0 \equiv P .
$$

The space $K\left(l_{2}\right)$ is complemented in its bidual (see [23]). Hence $\operatorname{Ext}\left(L_{1}, K\left(l_{2}\right)\right)$ $=0$ by the Lindenstrauss lifting principle (see [25, 23], but also [5]). Thus, the lower pull-back sequence in the diagram

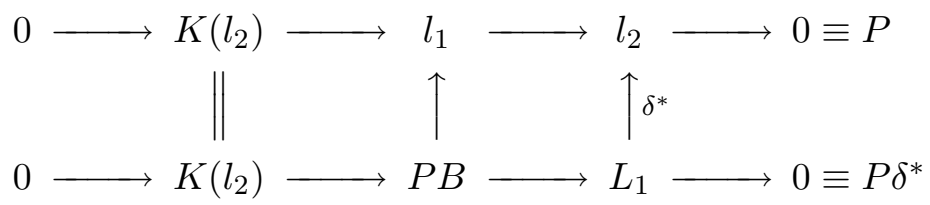

splits, and therefore the quotient map $\delta^{*}: L_{1} \rightarrow l_{2}$ can be lifted to $l_{1}$. In this way, there is an operator $\phi$ such that $P \equiv \phi F^{*}$ (see [10]) and $P$ is a push-out of $F^{*}$; by Lemma 2.1, it must also be $C(K)$-trivial. But if a projective presentation of $l_{2}$ is $C(K)$-trivial then $\operatorname{Ext}\left(l_{2}, C(K)\right)=0$, and it was proved by Kalton in [21] (see also [7]) that $\operatorname{Ext}\left(l_{2}, C[0,1]\right) \neq 0$.

The bidual sequence

$$
0 \rightarrow l_{2} \stackrel{\delta^{* *}}{\longrightarrow} C\left(B_{l_{2}}\right)^{* *} \rightarrow Q^{* *} \rightarrow 0 \equiv F^{* *}
$$


is also not $C(K)$-trivial: since $I=C\left(B_{l_{2}}\right)^{* *}$ is injective, it has the Grothendieck property (see $[12$, Cor. 12 , p. 156]) and thus every operator $I \rightarrow S$ into a separable space is weakly compact. By the Dunford-Pettis property of $I$, weakly compact operators on $I$ transform weakly compact sets onto norm compact sets; thus, the canonical inclusion $l_{2} \rightarrow C\left(B_{l_{2}}\right)$ cannot be extended to $I$. Another example is provided by sequences having the form

$$
0 \rightarrow c_{0}\left(A_{n}\right) \rightarrow c_{0}\left(l_{\infty}^{m(n)}\right) \rightarrow c_{0}\left(C_{n}\right) \rightarrow 0 \equiv F
$$

they have the property that $F$ and $F^{*}$ do $C(K)$-split although $F^{* *}$ does not necessarily $C(K)$-split.

3. On Banach spaces of Lindenstrauss-Pełczyński type. There is an obvious difference between the Lindenstrauss-Pełczyński [26] and the Johnson-Zippin [18] theorems. While the former asserts that every sequence $0 \rightarrow H \rightarrow c_{0} \rightarrow c_{0} / H \rightarrow 0 \equiv F$ is $C(K)$-trivial the latter establishes that the dual sequence $F^{*}$ is $\mathcal{L}_{\infty}$-trivial. Let us see that the class $\mathcal{L}_{\infty}$ cannot be enlarged, obtaining in this way a new characterization of $\mathcal{L}_{\infty}$-spaces.

Proposition 3.1. For a Banach space $E$ the following are equivalent:

(1) $E$ is an $\mathcal{L}_{\infty}$-space.

(2) Every E-valued operator defined on a $w\left(l_{1}, c_{0}\right)$-closed subspace of $l_{1}$ can be extended to $l_{1}$.

Proof. Recall that $w\left(l_{1}, c_{0}\right)$-closed subspaces of $l_{1}$ are precisely the orthogonal complements $H^{\perp}$ to subspaces $H$ of $c_{0}$. So, as we said in Section 1, (2) can be written as: $\operatorname{Ext}\left(H^{*}, E\right)=0$ for every subspace $H$ of $c_{0}$.

General structure results of Johnson-Rosenthal and Zippin (see [28, 1.g.2 and 2.d.1]) imply that given a subspace $H$ of $c_{0}$ there exist sequences $\left(A_{n}\right)$ and $\left(B_{n}\right)$ of finite-dimensional spaces such that there is an exact sequence $0 \rightarrow c_{0}\left(A_{n}\right) \rightarrow H \rightarrow c_{0}\left(B_{n}\right) \rightarrow 0$. Therefore, there is an exact sequence $0 \rightarrow l_{1}\left(B_{n}^{*}\right) \rightarrow H^{*} \rightarrow l_{1}\left(A_{n}^{*}\right) \rightarrow 0$. A simple 3 -space argument (see [6] or [7, Cor. 1.2]) then shows that $\operatorname{Ext}\left(H^{*}, E\right)=0$ is equivalent to $\operatorname{Ext}\left(l_{1}\left(A_{n}^{*}\right), E\right)=$ $0=\operatorname{Ext}\left(l_{1}\left(B_{n}^{*}\right), E\right)$. Therefore, $(2)$ is equivalent to $\operatorname{Ext}\left(l_{1}\left(G_{n}\right), E\right)=0$ for every sequence $\left(G_{n}\right)$ of finite-dimensional spaces. It was already observed by Johnson [14] that a sequence $0 \rightarrow Y \rightarrow X \rightarrow Z \rightarrow 0 \equiv F$ locally splits if and only if $F T \equiv 0$ for every operator $T: l_{1}\left(G_{n}\right) \rightarrow Z$ with $G_{n}$ finite-dimensional. Thus, every sequence $0 \rightarrow E \rightarrow X \rightarrow Z \rightarrow 0$ locally splits and in particular so does any sequence $0 \rightarrow E \rightarrow l_{\infty}(I) \rightarrow Q \rightarrow 0 \equiv G$. Recall from [20] that an exact sequence $F$ locally splits if and only if $F^{* *}$ splits. Hence, the bidual sequence $G^{* *}$ splits, $E^{* *}$ must be complemented in an $\mathcal{L}_{\infty}$-space, so it is an $\mathcal{L}_{\infty}$-space and $E$ must itself be an $\mathcal{L}_{\infty}$-space.

Not entirely trivial is the observation that condition (2) can be replaced by $\left(2^{\prime}\right)$ for every set $\Gamma$, every $E$-valued operator defined on a $w\left(l_{1}(\Gamma), c_{0}(\Gamma)\right)$ - 
closed subspace of $l_{1}(\Gamma)$ can be extended to $l_{1}(\Gamma)$. The proof only requires to take into consideration the decomposition lemma of [17].

The situation outlined for $w^{*}\left(l_{1}, c_{0}\right)$-closed subspaces of $l_{1}$, together with Lindenstrauss-Pełczyński's remark in [26] asserting that operators with ranges being isometric preduals of $L_{1}$ extend from subspaces of $c_{0}$ to the whole space, suggest investigating how much the class of $C(K)$-spaces can be enlarged in the Lindenstrauss-Pełczyński theorem.

Definition 2. We shall say that a Banach space $E$ is a LindenstraussPełczyński space, for short an $\mathcal{L} \mathcal{P}$-space, if all operators from subspaces of $c_{0}$ into $E$ can be extended to $c_{0}$.

We shall also need the quantitative version: when every operator $T$ : $H \rightarrow E$ admits an extension $\widehat{T}: c_{0} \rightarrow E$ such that $\|\widehat{T}\| \leq \lambda\|T\|$ we shall

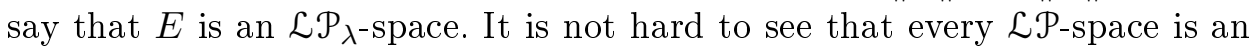

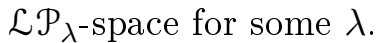

Lemma 3. An $\mathcal{L} \mathcal{P}_{\lambda}$-space is, for every $\varepsilon>0$, an $\mathcal{L}_{\infty, 2 \lambda+\varepsilon}$-space.

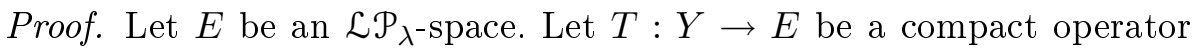
from a subspace $Y$ of a separable space $X$. Schauder's theorem ( $T$ is compact if and only if $T^{*}$ is compact) plus the characterization of compact sets in Banach spaces as subsets of the closed convex hull of a norm null sequence immediately show that $T$ factorizes through some subspace $i: H \rightarrow c_{0}$ as $T=B A$ with $A: Y \rightarrow H$ and $B: H \rightarrow E$. By definition, there is an extension $B_{1}: c_{0} \rightarrow E$ of $B$ with $\left\|B_{1}\right\| \leq \lambda\|B\|$; moreover Sobczyk's theorem gives an extension $A_{1}: X \rightarrow c_{0}$ of $i A$ with $\left\|A_{1}\right\| \leq 2\|i A\|$. The composition $B_{1} A_{1}: X \rightarrow E$ extends $T$ and satisfies $\left\|B_{1} A_{1}\right\| \leq 2 \lambda\|T\|$.

Let us now show that "if $E$-valued compact norm one operators can be extended with norm at most $c$ then for every $\varepsilon>0, E$ is an $\mathcal{L}_{\infty, c+\varepsilon^{-}}$space". To this end, let $\tau: Y \rightarrow E$ be any (compact or not) norm one operator. Each restriction $\tau_{F}: F \rightarrow E$ of $\tau$ to a finite-dimensional subspace $F$ of $Y$ is compact and can therefore be extended to an operator $T_{F}: X \rightarrow E$ with norm at most $c$. Define the operator $T: X \rightarrow E^{* *}$ by

$$
T(x)=w^{*}-\lim _{\mathcal{U}(F)} T_{F}(x)
$$

where $\mathcal{U}$ is a free ultrafilter on the set of finite-dimensional subspaces of $Y$ compatible with the natural ordering. This operator $T$ has norm at most $c$ and extends $\tau: Y \rightarrow E^{* *}$. In particular, given an exact sequence $0 \rightarrow$ $E \rightarrow V \rightarrow Q \rightarrow 0$, the canonical embedding $\delta: E \rightarrow E^{* *}$ can be extended to $V$ with norm at most $c$. So $E^{* *}$ is complemented in $V^{* *}$ with norm $c+\varepsilon$ (because of the principle of local reflexivity). Applying this to any embedding $0 \rightarrow E \rightarrow l_{\infty}(I) \rightarrow Q \rightarrow 0$ one finds that $E^{* *}$ is an $\mathcal{L}_{\infty, c+\varepsilon^{-s p a c e} \text {, as also }}$ is $E$. 
The above argument is modeled over Lindenstrauss' construction in [24]. A direct application of Lindenstrauss' result is awkward: as the referee of the paper remarked, in his memoir, Lindenstrauss considered what he calls $\mathcal{N}_{\lambda}$-spaces, which are spaces that are the closure of a directed union of finitedimensional $\lambda$-injective spaces; today we know that an $\mathcal{N}_{\lambda^{-}}$-space is an $\mathcal{L}_{\infty, \beta^{-}}$ space, but we do not know how $\beta$ depends on $\lambda$.

The converse fails: we show that not every $\mathcal{L}_{\infty}$-space is an $\mathcal{L} \mathcal{P}$-space. This solves Zippin's Problem 6.15 in [36]. The example (which was sketched in [8]) is based on the Bourgain-Pisier construction [3] which shows that for every separable Banach space $X$ there is an exact sequence

$$
0 \rightarrow X \rightarrow \mathcal{L}_{\infty}(X) \rightarrow \mathcal{L}_{\infty}(X) / X \rightarrow 0 \equiv \mathrm{BP}_{X},
$$

in which $\mathcal{L}_{\infty}(X)$ is a separable $\mathcal{L}_{\infty}$-space and $\mathcal{L}_{\infty}(X) / X$ has the Schur property (weakly convergent sequences are norm convergent).

Proposition 3.2. Let $H$ be a subspace of $c_{0}$ such that $c_{0} / H$ is not isomorphic to $c_{0}$. Then $\mathcal{L}_{\infty}(H)$ is not an $\mathcal{L} \mathcal{P}$-space. In fact, there exists an operator $H \rightarrow \mathcal{L}_{\infty}(H)$ which does not extend to $c_{0}$.

Proof. Consider the sequence $0 \rightarrow H \stackrel{j}{\rightarrow} c_{0} \rightarrow c_{0} / H \rightarrow 0 \equiv F$. Let $0 \rightarrow$ $H \stackrel{i}{\rightarrow} \mathcal{L}_{\infty}(H) \rightarrow S \rightarrow 0 \equiv \mathrm{BP}_{H}$ be a Bourgain-Pisier sequence associated with $H$. Assume that $i$ extends to $c_{0}$ through $j$, so that $F$ is a pull-back of $\mathrm{BP}_{H}$. By Sobczyk's theorem [28, 2.f.5], $\mathrm{BP}_{H}$ is a pull-back of $F$. Applying the diagonal principle [27, Thm. 2] one gets an isomorphism

$$
\mathcal{L}_{\infty}(H) \oplus c_{0} / H \simeq c_{0} \oplus S .
$$

In particular, $c_{0} / H$ is a complemented subspace of $c_{0} \oplus S$. Since $S$ and $c_{0}$ are totally incomparable by the Schur property of $S$ the decomposition theorem of Edelstein-Wojtaszczyk (see [28, Thm. 2.c.13]) ensures that $c_{0} / H$ is isomorphic to some $A \oplus B$ with $A$ complemented in $c_{0}$ and $B$ complemented in $S$. Since $c_{0} / H$ is a subspace of $c_{0}[28,2$.f.6], $B$ can only be finite-dimensional, hence $c_{0} / H \simeq c_{0}$, contrary to hypothesis.

This example immediately implies

EXAMPLE. The $c_{0}$-amalgam of $\left(\mu, \mathcal{L}_{\infty}\right)$-trivial sequences is not necessarily $\mathcal{L}_{\infty}$-trivial.

Proof. As already mentioned, given a subspace $H$ of $c_{0}$ there exist sequences $\left(A_{n}\right)$ and $\left(B_{n}\right)$ of finite-dimensional spaces such that there is an exact sequence $0 \rightarrow c_{0}\left(A_{n}\right) \rightarrow H \rightarrow c_{0}\left(B_{n}\right) \rightarrow 0$. Clearly, the exact sequences $0 \rightarrow A_{n} \rightarrow l_{\infty}^{m(n)} \rightarrow C_{n} \rightarrow 0$ are $\left(\lambda, \mathcal{L}_{\infty, \lambda}\right)$-trivial. If all the amalgams $0 \rightarrow c_{0}\left(A_{n}\right) \rightarrow c_{0}\left(l_{\infty}^{m(n)}\right) \rightarrow c_{0}\left(C_{n}\right) \rightarrow 0$ were $\mathcal{L}_{\infty}$-trivial then every sequence $0 \rightarrow H \rightarrow c_{0} \rightarrow c_{0} / H \rightarrow 0$ would also be $\mathcal{L}_{\infty}$-trivial: indeed, there 
would be a complete push-out diagram

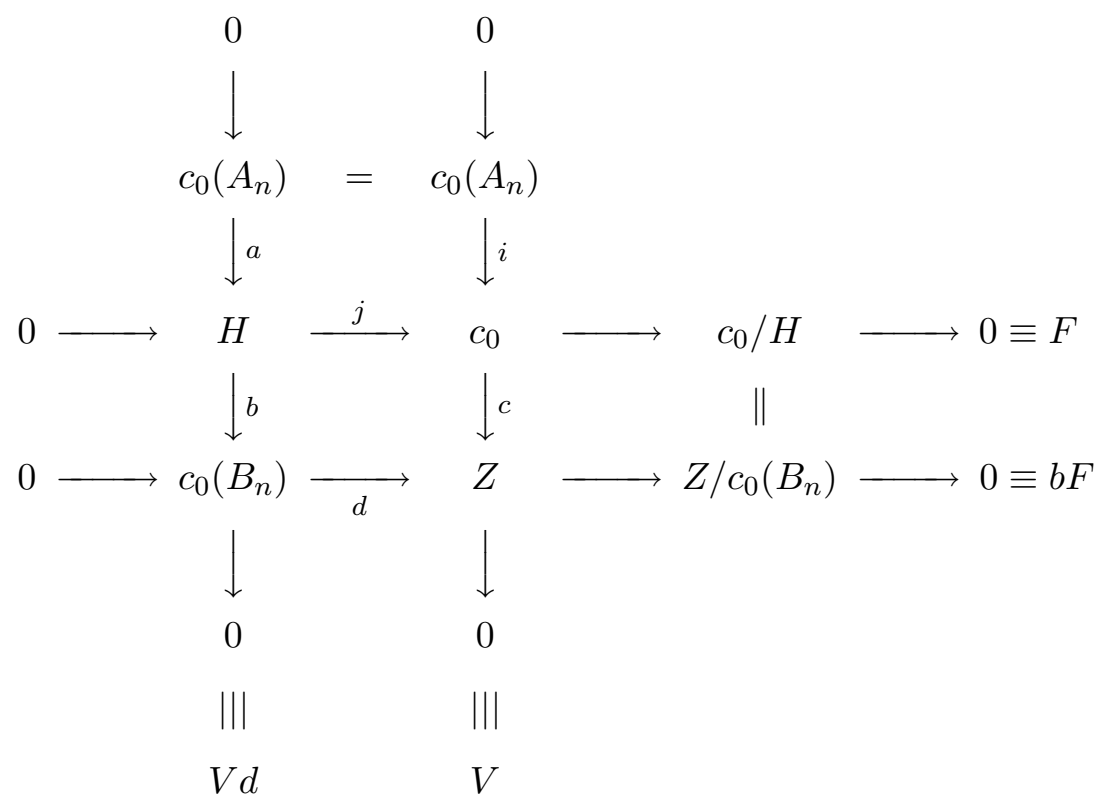

to which we apply Lemma $1(\mathrm{i})$ : if $V$ and $b F$ are $\mathcal{L}_{\infty}$-trivial then $F$ is $\mathcal{L}_{\infty}$-trivial (which we know it is not). It remains to check that $V$ and $b F$ are $\mathcal{L}_{\infty}$-trivial. The sequence $V$ is $\mathcal{L}_{\infty}$-trivial by our assumption and the Lindenstrauss-Rosenthal theorem that asserts that $c_{0}$ is automorphic (see $[27,10])$; i.e., there exists an isomorphism $\tau: c_{0} \rightarrow c_{0}$ making the diagram

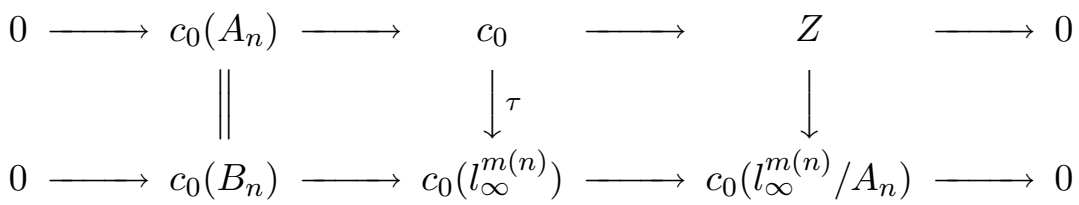

commutative and therefore the two sequences $\mathcal{L}_{\infty}$-split simultaneously. The sequence $b F$ is $\mathcal{L}_{\infty}$-trivial by essentially the same arguments taking into account that $Z$ must be a subspace of $c_{0}$.

The problem of identifying $\mathcal{L} \mathcal{P}$-spaces is still far from being solved, and it actually gives rise to interesting questions. Observe that, in addition to $C(K)$-spaces, it is clear that complemented subspaces of $C(K)$-spaces and separably injective spaces are also $\mathcal{L} \mathcal{P}$-spaces. The reader might be surprised by the distinction between the two, especially regarding the fact that every injective space is complemented in some $C(K)$-space. Let us show that the two classes are indeed distinct.

Proposition 3.3. There exists a separably injective space that is not complemented in any $C(K)$-space. 
Proof. Consider the pull-back diagram

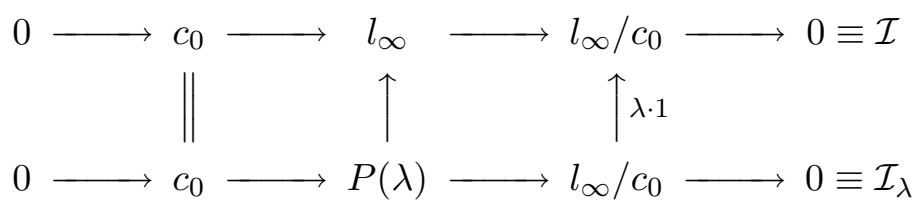

Benyamini shows in [1] that $P(\lambda)$ is no less than $\lambda^{-1}$ complemented in any $C(K)$-space. Thus, the $c_{0}$-amalgam of the family $\left(\mathcal{I}_{n^{-1}}\right)$

$$
0 \rightarrow c_{0}\left(c_{0}\right) \rightarrow c_{0}\left(P\left(n^{-1}\right)\right) \rightarrow c_{0}\left(l_{\infty} / c_{0}\right) \rightarrow 0 \equiv c_{0}\left(\mathcal{I}_{n^{-1}}\right)
$$

provides an exact sequence in which both $c_{0}\left(c_{0}\right)$ as well as $c_{0}\left(l_{\infty} / c_{0}\right)$ are $C(K)$-spaces. However, the space $c_{0}\left(P\left(n^{-1}\right)\right)$ cannot be complemented in any $C(K)$-space. That $c_{0}$ is separably injective is precisely Sobczyk's theorem. That $l_{\infty} / c_{0}$ is separably injective is well known and follows from Proposition 4.3 below. It was shown in $[15,32]$ that when $X$ is separably injective then $c_{0}(X)$ is separably injective as well. Finally, separable injectivity is a 3 -space property [6].

There are other $\mathcal{L} \mathcal{P}$-spaces. As mentioned before, according to [26, p. 234, Remark 2], isometric preduals of $L_{1}$ are $\mathcal{L} \mathcal{P}$-spaces. So, it is quite natural to ask whether the previous classes (namely: complemented subspaces of a $C(K)$-space, separably injective spaces and isometric preduals of $L_{1}$ ) exhaust the $\mathcal{L} \mathcal{P}$-spaces. The answer is no.

\section{Proposition 3.4. Every $\mathcal{L}_{\infty}$-space not containing $c_{0}$ is an $\mathcal{L} \mathcal{P}$-space.}

Proof. Let $X$ be a Banach space. If $T$ is a noncompact operator whose domain is a subspace of $c_{0}$ then $T$ is an isomorphism onto a copy of $c_{0}$; hence, when $Y$ contains no copy of $c_{0}$ every operator $H \rightarrow Y$ must be compact. Now Lindenstrauss' extension theorem for compact operators [24] yields the result.

By a result of Johnson and Zippin [16] separable isometric $L_{1}$-preduals are quotients of $C[0,1]$. Observe that $\mathcal{L}_{\infty}$-spaces not containing $c_{0}$ cannot be quotients of $C(K)$-spaces (since, by [29], every operator on a $C(K)$-space is either weakly compact or an isomorphism onto a copy of $c_{0}$ ). Concrete examples of $\mathcal{L}_{\infty}$-spaces not containing $c_{0}$ can be obtained by applying the Bourgain-Pisier construction $0 \rightarrow X \rightarrow \mathcal{L}_{\infty}(X) \rightarrow S \rightarrow 0$ to spaces $X$ without copies of $c_{0}$ by a simple 3 -space argument (see [9, Thm. 3.2.e]).

New examples of $\mathcal{L} \mathcal{P}$-spaces can be obtained by showing that this class has the 3 -space property.

4. The 3-space problem for $\mathcal{L} \mathcal{P}$-spaces. The purpose of this section is to show:

TheOREM 2. The class of $\mathcal{L} \mathcal{P}$-spaces has the 3 -space property. 
The proof is not simple and requires both a different characterization of $\mathcal{L} \mathcal{P}$-spaces and a new method to obtain 3 -space properties. We assume from the reader some acquaintance with the theory of operator ideals as developed by Pietsch in [31]. Recall that an operator ideal $\mathfrak{A}$ is said to be surjective (see $[31,4.7 .9])$ if whenever $Q$ is a quotient map and $T Q \in \mathfrak{A}$ then $T \in \mathfrak{A}$; dually, the ideal $\mathfrak{A}$ is injective (see [31, 4.6.9]) if whenever $J$ is an into isomorphism and $J T \in \mathfrak{A}$ then $T \in \mathfrak{A}$. Consider the operator ideal $\mathfrak{J}_{0}$ of those operators that factorize through a subspace of $c_{0}$. Recall that a functor is called exact when it transforms exact sequences into exact sequences.

Proposition 4.1. A Banach space $E$ is an $\mathcal{L} \mathcal{P}$-space if and only if the functor $\mathfrak{J}_{0}(\cdot, E)$ is exact when acting on the category of separable Banach spaces.

Proof. Let $j: Y \rightarrow X$ be an into isomorphism, and let $T \in \mathfrak{J}_{0}(Y, E)$. Write $T=R S$ with $S \in \mathfrak{L}(Y, H), R \in \mathfrak{L}(H, E)$ and let $i: H \rightarrow c_{0}$ be an into isomorphism. The operator $R$ can be extended to an operator $R_{1}: c_{0} \rightarrow E$ through $i$ since $E$ is an $\mathcal{L} \mathcal{P}$-space; moreover Sobczyk's theorem allows one to extend $i S: Y \rightarrow c_{0}$ to an operator $S_{1}: X \rightarrow c_{0}$ through $j$. The operator $R_{1} S_{1}$ is an extension of $T$ through $j$. The other implication is immediate.

The new method to obtain 3 -space properties is the following.

Proposition 4.2. Let $\mathfrak{A}$ be a surjective and injective operator ideal. The class of all Banach spaces $E$ such that the functor $\mathfrak{A}(\cdot, E)$ is exact has the 3-space property.

Proof. The surjectivity of $\mathfrak{A}$ implies that given an exact sequence $0 \rightarrow$ $Y \stackrel{j}{\rightarrow} X \stackrel{q}{\rightarrow} Z \rightarrow 0$ and a space $E$ the induced sequence

$$
0 \rightarrow \mathfrak{A}(Z, E) \stackrel{q^{*}}{\rightarrow} \mathfrak{A}(X, E) \stackrel{j^{*}}{\rightarrow} \mathfrak{A}(Y, E)
$$

is exact. Now, let

$$
0 \rightarrow A \stackrel{i}{\rightarrow} B \stackrel{p}{\rightarrow} C \rightarrow 0
$$

be an exact sequence. By assumption, both $\mathfrak{A}(\cdot, A)$ and $\mathfrak{A}(\cdot, C)$ are exact functors and we need to prove that also $\mathfrak{A}(\cdot, B)$ is exact. To this end we construct the commutative diagram

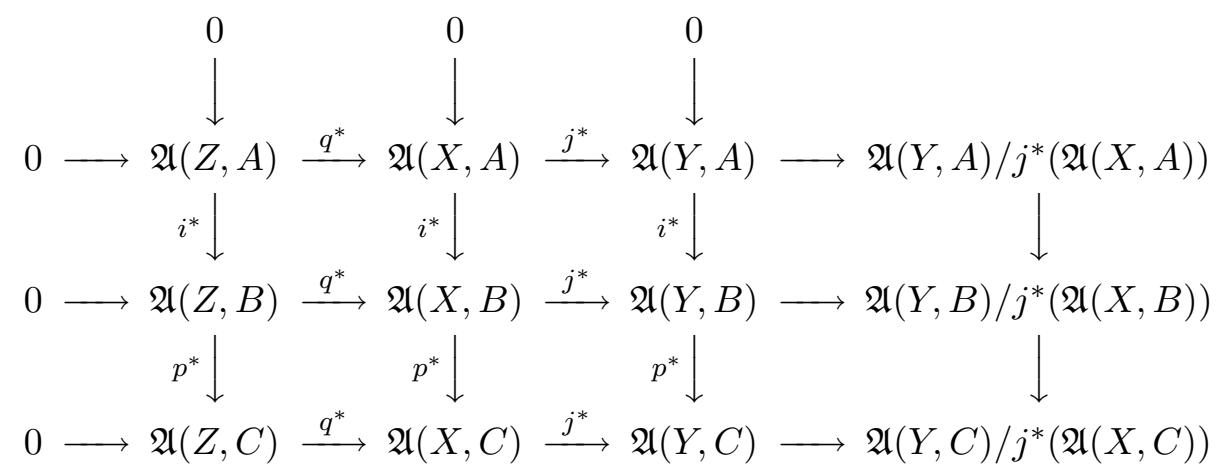


The rows are exact by the surjectivity of $\mathfrak{U}$, while the columns are also exact by injectivity of $\mathfrak{U}$. By hypothesis,

$$
\mathfrak{A}(Y, A) / j^{*}(\mathfrak{A}(X, A))=\mathfrak{A}(Y, C) / j^{*}(\mathfrak{A}(X, C))=0
$$

and the exactness of the fourth column implies that

$$
\mathfrak{A}(Y, B) / j^{*}(\mathfrak{A}(X, B))=0,
$$

hence $\mathfrak{A}(\cdot, B)$ is exact.

Since the separability assumption of the previous characterization of $\mathcal{L} \mathcal{P}$ spaces does not affect the method of Proposition 4.2, the proof of Theorem 2 will be complete after showing:

Lemma 1. The ideal $\mathfrak{J}_{0}$ is injective and surjective.

Proof. The injectivity is a direct consequence of the definition. To show the surjectivity, let $\tau: X \rightarrow E$ be an operator which factorizes as $\tau=\varphi_{0} \varphi_{1}$ through a subspace $H$ of $c_{0}$ in a diagram

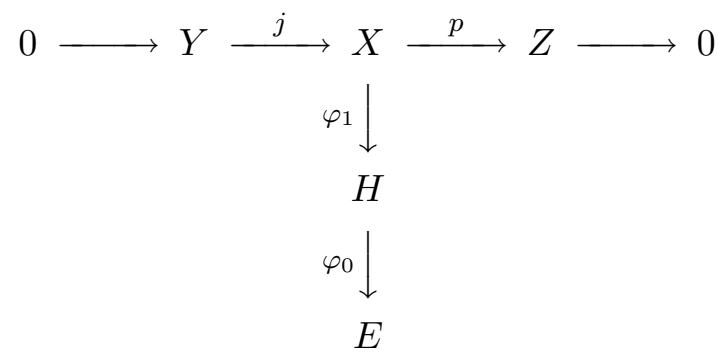

Assume that $\tau j=0$. One then has the commutative diagram

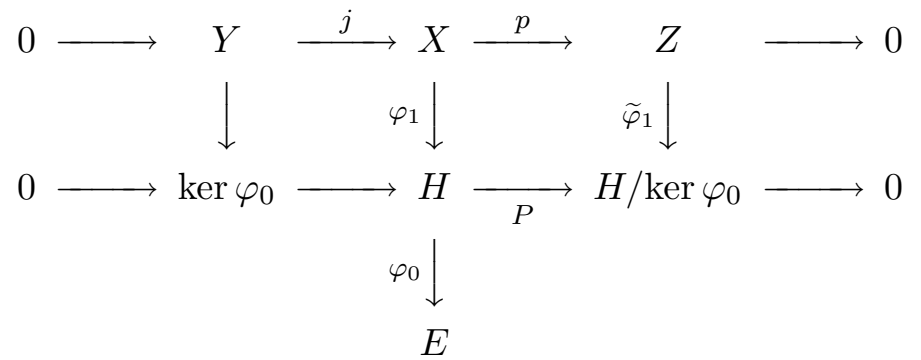

It is clear that there exists an operator $\widetilde{\varphi}_{0}: H / \operatorname{ker} \varphi_{0} \rightarrow E$ such that $\widetilde{\varphi}_{0} P=\varphi_{0}$. It is then obvious that $\widetilde{\varphi}_{0} \widetilde{\varphi}_{1} p=\varphi_{0} \varphi_{1}$. Moreover the operator $\widetilde{\varphi}_{0} \widetilde{\varphi}_{1}$ is in $\mathfrak{I}_{0}(Z, E)$ since $H / \operatorname{ker} \varphi_{0}$, as a quotient of a subspace of $c_{0}$, is itself a subspace of a quotient of $c_{0}$, hence [28, 2.f.6] a subspace of $c_{0}$.

Theorem 2, in particular, yields:

Corollary 1. Every twisted sum of $C(K)$-spaces is an $\mathcal{L} \mathcal{P}$-space.

The paper [7] contains most of the available information about how to construct twisted sums of $C(K)$-spaces. For instance, it is shown that for 
every separable Banach space $X$ not containing $l_{1}$ there exists an exact sequence

$$
0 \rightarrow C[0,1] \stackrel{i}{\rightarrow} \Omega(X) \stackrel{q}{\rightarrow} X \rightarrow 0
$$

with strictly singular quotient map. Of course, the space $\Omega(X)$ is not a quotient of a $C(K)$-space. Using Theorem 4.7 of [7] one can obtain examples of $\mathcal{L} \mathcal{P}$-spaces not containing $l_{1}$ which are not $C(K)$-spaces. We do not know if there exist $\mathcal{L}_{\infty}$-spaces not containing $l_{1}$ which are not $\mathcal{L} \mathcal{P}$-spaces.

New nonseparable $\mathcal{L} \mathcal{P}$-spaces can be obtained through the following stability result.

Proposition 4.3. Every quotient of an $\mathcal{L} \mathcal{P}$-space by a separably injective space is an $\mathcal{L} \mathcal{P}$-space.

Proof. Consider an exact sequence $0 \rightarrow$ SI $\rightarrow \mathcal{L} \mathcal{P} \stackrel{q}{\rightarrow} Q \rightarrow 0$ in which the middle term is a Lindenstrauss-Pełczyński space and the subspace is separably injective. Let $\phi: H \rightarrow Q$ be an operator from a subspace $H$ of $c_{0}$. Since SI is separably injective, $\operatorname{Ext}(H, \mathrm{SI})=0$. Hence $F \phi$ splits and $\phi$ can be lifted through $q$ to an operator $\psi: H \rightarrow \mathcal{L} \mathcal{P}$. This operator can be extended to an operator $\Psi: c_{0} \rightarrow \mathcal{L} \mathcal{P}$. The operator $q \Psi: c_{0} \rightarrow Q$ is the desired extension of $\phi$.

The same proof shows that the quotient of two separably injective spaces is separably injective. In particular, $l_{\infty} / c_{0}$ is separably injective. However, the following example shows that the quotient of two $\mathcal{L} \mathcal{P}$-spaces is not necessarily an $\mathcal{L} \mathcal{P}$-space.

Proposition 4.4. Every separable $\mathcal{L}_{\infty}$-space is a quotient of two $\mathcal{L} \mathcal{P}_{-}$ spaces.

Proof. Let $X$ be a separable $\mathcal{L}_{\infty}$-space. Consider the following push-out diagram:

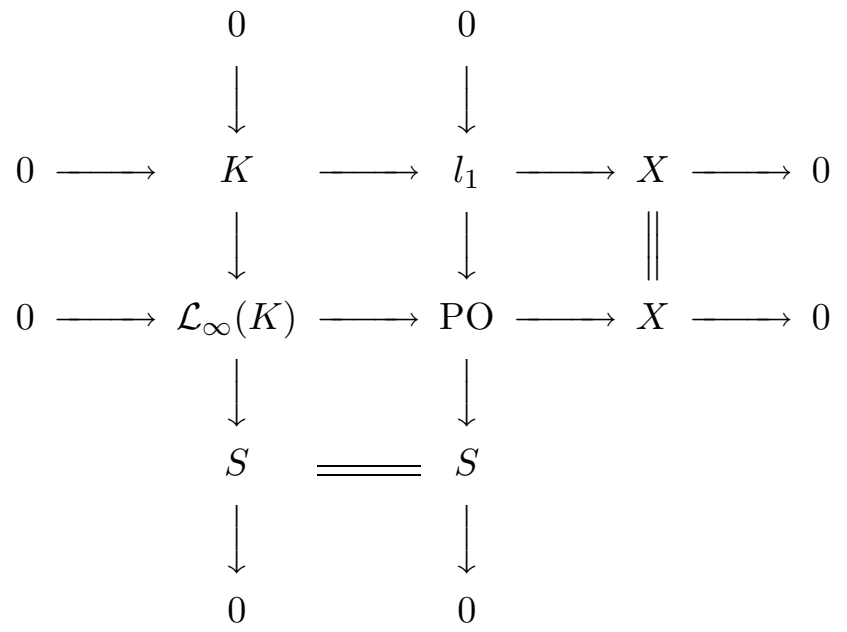


The push-out space $\mathrm{PO}$ is an $\mathcal{L}_{\infty}$-space by a simple 3 -space argument (see $[9$, Thm. 3.3.b]). Since the Schur property is also a 3 -space property (see [9, 6.1]), both $\mathcal{L}_{\infty}(K)$ and PO are therefore $\mathcal{L}_{\infty}$-spaces with the Schur property. Thus, they are both $\mathcal{L} \mathcal{P}$-spaces, while their quotient is $X$.

Corollary 2. Every separable $\mathcal{L}_{\infty}$-space is a quotient of two $\mathcal{L}_{\infty}$-spaces with the Schur property.

Therefore, the choice $X=\mathcal{L}_{\infty}(H)$ of a separable $\mathcal{L}_{\infty}$-space that is not an $\mathcal{L} \mathcal{P}$-space, as the one constructed in Proposition 3.2, shows that the quotient of two $\mathcal{L} \mathcal{P}$-spaces need not be an $\mathcal{L} \mathcal{P}$-space, and similarly for the quotient of two $\mathcal{L}_{\infty}$-spaces with the Schur property.

5. Some open questions. We have already shown that the quotient of two $\mathcal{L} \mathcal{P}$-spaces is not necessarily an $\mathcal{L} \mathcal{P}$-space. An especially interesting case is:

Question 1 . Is $l_{\infty} / C[0,1]$ an $\mathcal{L} \mathcal{P}$-space?

A variation of this question is:

Question 2. Must $\mathcal{L}_{\infty}$-spaces which are quotients of $C[0,1]$ be $\mathcal{L} \mathcal{P}_{-}$ spaces?

As mentioned in the introduction, Johnson and Zippin proved in [17] that every extension $0 \rightarrow H \rightarrow c_{0}(\Gamma) \rightarrow Z \rightarrow 0$ is $C(K)$-trivial. It would be nice to know if $\mathcal{L} \mathcal{P}$-spaces can play the role of $C(K)$-spaces in this result.

Question 3. Given a subspace $H$ of $c_{0}(\Gamma)$, does every operator $H \rightarrow \mathcal{L} \mathcal{P}$ have an extension to $c_{0}(\Gamma)$ ?

Needless to say, the extension property one would like to get from $\mathcal{L} \mathcal{P}$ spaces is: every $C(K)$-trivial sequence is also $\mathcal{L} \mathcal{P}$-trivial. Unfortunately, this does not hold.

ExAMPLE. We already know that the Bourgain-Pisier space $\mathcal{L}_{\infty}\left(l_{2}\right)$ is an $\mathcal{L} \mathcal{P}$-space. Consider then the sequences

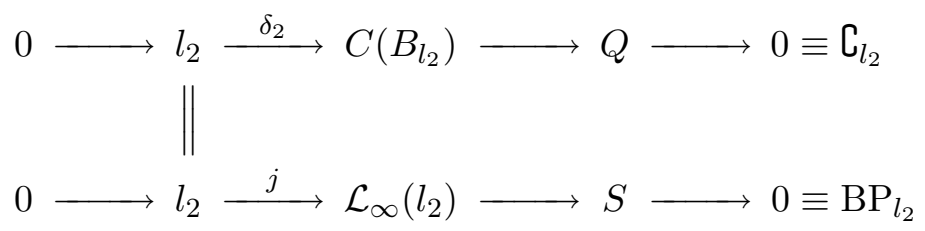

If $j$ could be extended to an operator $J: C\left(B_{l_{2}}\right) \rightarrow \mathcal{L}_{\infty}\left(l_{2}\right)$ through $\delta_{2}$ this would be a weakly compact operator since $\mathcal{L}_{\infty}\left(l_{2}\right)$ does not contain $c_{0}$. Hence $J$ would be completely continuous by the Dunford-Pettis property of $C(K)$-spaces. It is therefore impossible that $J \delta_{2}=j$. 
The method of proof developed in Proposition 4.2 is new. It is moreover clear that it can be applied to other injective and surjective operator ideals appearing in the literature. It is easy to check that the following ideals are injective and surjective (see also [31]): $\mathfrak{L}=$ all operators; $\mathfrak{F}=$ finite rank operators; $\mathfrak{K}=$ compact operators; $\mathfrak{W}=$ weakly compact operators; $\mathfrak{U}=$ unconditionally summing operators; $\mathfrak{J}_{2}=$ operators factorable through a Hilbert space. Let us introduce some notation: given an injective and surjective operator ideal $\mathfrak{U}$ let $E(\mathfrak{U})$ be the class of all Banach spaces $E$ such that the functor $\mathfrak{U}(\cdot, E)$ is exact. From Proposition 4.2 we have obtained easy proofs that the following classes have the 3-space property: $E(\mathfrak{L})=$ injective spaces; applying the method only to separable spaces one obtains the class of separably injective spaces; $E(\mathfrak{K})=\mathcal{L}_{\infty}$-spaces (by [24]); $E(\mathfrak{W})$ $=\mathfrak{L}_{\infty}$-spaces with the Schur property (shown in [2]). The classes $E(\mathfrak{U})$ and $E\left(\mathfrak{J}_{2}\right)$ seem not to have been characterized yet.

A simple homological duality argument yields:

Proposition 5.1. Let $\mathfrak{A}$ be an injective and surjective operator ideal. The class of all Banach spaces $E$ such that the functor $\mathfrak{A}(E, \cdot)$ is exact has the 3-space property.

If we write $\exists(\mathfrak{A})$ for the previous class determined by the ideal $\mathfrak{A}$ then the only nontrivial case identified is $\exists(\mathfrak{K})=\mathcal{L}_{1}$-spaces.

\section{References}

[1] Y. Benyamini, An M-space which is not isomorphic to a $C(K)$-space, Israel J. Math. 28 (1977), 98-102.

[2] J. Bourgain and F. Delbaen, A class of special $\mathcal{L}_{\infty}$-spaces, Acta Math. 145 (1980), $155-176$.

[3] J. Bourgain and G. Pisier, A construction of $\mathcal{L}_{\infty}$-spaces and related Banach spaces, Bol. Soc. Brasil. Mat. 14 (1983), 109-123.

[4] F. Cabello Sánchez and J. M. F. Castillo, Duality and twisted sums of Banach spaces, J. Funct. Anal. 175 (2000), 1-16.

[5] - - - Uniform boundedness and twisted sums of Banach spaces, Houston J. Math. 30 (2004), 523-536.

[6] - - - The long homology sequence for quasi-Banach spaces, with applications, Positivity 8 (2004), 379-394.

[7] F. Cabello Sánchez, J. M. F. Castillo, N. J. Kalton and D. T. Yost, Twisted sums with $C(K)$ spaces, Trans. Amer. Math. Soc. 355 (2003), 4523-4541.

[8] F. Cabello Sánchez, J. M. F. Castillo, Y. Moreno and D. Yost, Extension of $\mathcal{L}_{\infty}$ spaces under a twisted light, in: Proc. Internat. Conf. dedicated to the 110th anniversary of Stefan Banach, Lviv, V. Kadets and W. Żelazko (eds.), North-Holland Math. Stud.

[9] J. M. F. Castillo and M. González, Three-Space Problems in Banach Space Theory, Lecture Notes in Math. 1667, Springer, 1997. 
[10] J. M. F. Castillo and Y. Moreno, On the Lindenstrauss-Rosenthal theorem, Israel J. Math. 140 (2004), 253-270.

[11] J. Diestel, H. Jarchow and A. Tonge, Absolutely Summing Operators, Cambridge Stud. Adv. Math. 43, Cambridge Univ. Press, 1995.

[12] J. Diestel and J. J. Uhl, Jr., Vector Measures, Math. Surveys 15, Amer. Math. Soc., 1977.

[13] E. Hilton and K. Stammbach, A Course in Homological Algebra, Grad. Texts in Math. 4, Springer, 1970.

[14] W. B. Johnson, Extensions of $c_{0}$, Positivity 1 (1997), 55-74.

[15] W. B. Johnson and T. Oikhberg, Separable lifting property and extensions of local reflexivity, Illinois J. Math. 45 (2001), 123-137.

[16] W. B. Johnson and M. Zippin, Separable $L_{1}$ preduals are quotients of $C(\Delta)$, Israel J. Math. 16 (1973), 198-202.

[17] - - Extension of operators from subspaces of $c_{0}(\Gamma)$ into $C(K)$ spaces, Proc. Amer. Math. Soc. 107 (1989), 751-754.

[18] - - - Extension of operators from weak* -closed subspaces of $l_{1}$ into $C(K)$ spaces, Studia Math. 117 (1995), 43-55.

[19] N. J. Kalton, The three-space problem for locally bounded F-spaces, Compos. Math. 37 (1978), 243-276.

[20] - Locally complemented subspaces and $\mathcal{L}_{p}$ for $p<1$, Math. Nachr. 115 (1984), 71-97.

[21] - On subspaces of $c_{0}$ and extension of operators into $C(K)$-spaces, Quart. J. Math. Oxford 52 (2001), 313-328.

[22] N. J. Kalton and N. T. Peck, Twisted sums of sequence spaces and the three-space problem, Trans. Amer. Math. Soc. 255 (1979), 1-30.

[23] N. J. Kalton and A. Pełczyński, Kernels of surjections from $\mathcal{L}_{1}$-spaces with an application to Sidon sets, Math. Ann. 309 (1997), 135-158.

[24] J. Lindenstrauss, On the extension of compact operators, Mem. Amer. Math. Soc. 48 (1964).

[25] -, On a certain subspace of $l_{1}$, Bull. Polon. Acad. Sci. 12 (1964), 539-542.

[26] J. Lindenstrauss and A. Pełczyński, Contributions to the theory of the classical Banach spaces, J. Funct. Anal. 8 (1971), 225-249.

[27] J. Lindenstrauss and H. P. Rosenthal, Automorphisms in $c_{0}, l_{1}$ and $m$, Israel J. Math. 9 (1969), 227-239.

[28] J. Lindenstrauss and L. Tzafriri, Classical Banach Spaces I. Sequence Spaces, Ergeb. Math. Grenzgeb. 92, Springer, 1977.

[29] A. Pełczyński, Banach spaces on which every unconditionally converging operator is weakly compact, Bull. Polish Acad. Sci. 10 (1962), 641-648.

[30] - Linear extensions, linear averagings, and their applications to linear topological classification of spaces of continuous functions, Dissertationes Math. 58 (1968).

[31] A. Pietsch, Operator Ideals, North-Holland Math. Library 20, North-Holland, 1980.

[32] H. P. Rosenthal, The complete separable extension problem, J. Operator Theory 43 (2000), 329-374.

[33] M. Zippin, The embedding of Banach spaces into spaces with structure, Illinois J. Math. 34 (1990), 586-606.

[34] - , A global approach to certain operator extension problems, in: Lecture Notes in Math. 1470, Springer, 1990, 78-84.

[35] -, Applications of Michael's continuous selection theorem to operator extension problems, Proc. Amer. Math. Soc. 127 (1999), 1371-1378. 
[36] M. Zippin, Extension of bounded linear operators, in: Handbook of the Geometry of Banach spaces, Vol. 2, W. B. Johnson and J. Lindenstrauss (eds.), Elsevier, 2003, 1703-1741.

Departamento de Matemáticas

Universidad de Extremadura

Avenida de Elvas

06071 Badajoz, Spain

E-mail: castillo@unex.es

ymoreno@unex.es

jesus.suarez@math.uni-karlsruhe.de

Received March 22, 2004

Revised version January 7, 2006 